\title{
Population dynamics of mutualism and intraspecific density dependence: how $\theta$-logistic density dependence affects mutualistic positive feedback
}

\author{
Christopher M. Moore ${ }^{\mathrm{b}, *}$, Samantha A. Catella ${ }^{1}$, Karen C. Abbott ${ }^{1}$ \\ ${ }^{a}$ Department of Biology, Case Western Reserve University, Cleveland, OH 44106, United States \\ ${ }^{b}$ Beginning fall 2017: Department of Biology, Colby College, Waterville, ME 04901, United States
}

\begin{abstract}
Mutualism describes the biological phenomenon where two or more species are reciprocally beneficial, regardless of their ecological intimacy or evolutionary history. Classic theory shows that mutualistic benefit must be relatively weak, or else it overpowers the stabilizing influence of intraspecific competition and leads to unrealistic, unbounded population growth. Interestingly, the conclusion that strong positive interactions lead to runaway population growth is strongly grounded in the behavior of a single model. This model - the Lotka-Volterra competition model with a sign change to generate mutualism rather than competition between species - assumes logistic growth of each species plus a linear interaction term to represent the mutualism. While it is commonly held that the linear interaction term is to blame for the model's unrealistic behavior, we show here that a linear mutualism added to a $\theta$-logistic model of population growth can prevent unbounded growth. We find that when density dependence is decelerating, the benefit of mutualism at equilibrium is greater than when density dependence is accelerating. Although there is a greater benefit, however, decelerating density dependence tends to destabilize populations whereas accelerating density dependence is always stable. We interpret these findings tentatively, but with promise for the understanding of the population ecology of mutualism by generating several predictions relating growth rates of mutualist populations and the strength of mutualistic interaction.
\end{abstract}

Keywords: Mutualism, population dynamics, density dependence, Lotka-Volterra, $\theta$-logistic

\section{Introduction}

2 Mutualistic interactions describe the ecology of two or more species that reciprocally increase each 3 other's fitness (Bronstein, 2015). These interactions are arguably the most common type of ecological 4 interaction, and they have profoundly shaped biodiversity as we understand it. Examples include

\footnotetext{
* Corresponding author

Email address: christopher.moore@colby.edu (Christopher M. Moore)
} 
mutualisms between mycorrhizae and plants (van der Heijden et al., 2015), coral and zooxanthellae (Baker, 2003), plants and pollinators (Willmer, 2011), tending ants and aphids or Lepidoptera larvae (Rico-Gray and Oliveira, 2007; Stadler and Dixon, 2008), plants and seed-dispersing animals (Howe and Smallwood, 1982 Levey et al., 2002), lichens (fungi and algae) (Brodo et al., 2001), and plants and rhizobia (Sprent et al., 1987; Kiers et al. 2003). Despite mutualism's obvious importance, it was not until the latter part of the $20^{\text {th }}$ century that the natural history of mutualism underwent rigorous ecological study, the conceptual framework for mutualism was laid, and mutualism was no longer confounded with the concept of symbiosis. Thus, by the time mutualism was fully introduced to the larger ecological community, theoretical ecology had been developing in its absence for decades. This resulted in the paucity of theory for mutualisms still very much visible today.

Gause and Witt (1935) first used the Lotka-Volterra model of interspecific competition to investigate the case of facultative "mutual aid" between two species by reversing the sign of the linear competition term from negative to positive. They noted that with enough "mutual aid" the zerogrowth isoclines no longer cross to give a positive equilibrium point and species grow exponentially without bound - a biologically unrealistic scenario. More specifically, they found that if the product of the strength of mutualism between the two species is $\geq$ the product of the strength of intraspecific competition for each species, then the positive feedback of mutualism would overpower the negative feedback of intraspecific competition, resulting in unbounded growth. Following this pioneering study, no development of theory around mutualism would happen for over 30 years and ecologists were left lacking a basic theoretical explanation for what stabilizes mutualism in nature.

A key feature of the Lotka-Volterra model is its use of a linear functional response: the effect of a mutualist on its partner's per capita growth rate is linearly proportional to the mutualist's density. Early models of obligate mutualism also shared this feature. Albrecht et al. (1974), May (1976), Christiansen and Fenchel (1977), and Vandermeer and Boucher (1978) introduced the idea of modeling mutualism through the intrinsic growth rate, shifting it from positive, in the case of facultative mutualism, to negative for obligate mutualism. Using linear functional responses, they generally found that, first, two obligate mutualists cannot stably coexist and, second, stable coexistence is possible if one species is obligate and the other is not, depending on the strength of the mutualism. These papers and others (e.g, Wolin, 1985, DeAngelis et al., 1986) further postulated that mutualistic functional responses are nonlinear, and thus attributed the unrealistic behavior of the Lotka-Volterra and similar models to their use of a linear functional response. Nonlinear functional responses were later explicitly modeled (e.g., Wright, 1989; Holland et al., 2002, Holland and DeAngelis, 2010, Revilla, 2015), confirming that nonlinear functional responses can indeed 
stabilize mutualistic populations.

Each of the aforementioned mutualism models, regardless of the functional response, assumes linear intraspecific density dependence; i.e., logistic within-species dynamics. However, nonlinear density dependence has been observed in controlled laboratory populations of organisms with simple life histories, such as Daphnia sp. and other Cladocera (Smith, 1963 Smith and Cooper, 1982) and Drosophila spp. (Ayala et al., 1973 Gilpin and Ayala, 1973, Pomerantz et al., 1980), and in long-term datasets on species with more complex life histories (Stubbs, 1977, Fowler, 1981; Sibly et al., 2005 Coulson et al. 2008). Models that relax the assumption of linear intraspecific density dependence have been proposed for single species (e.g., Richards, 1959, Schoener, 1973, Turchin, 2003 , Sibly et al. 2005) and communities with two or more competitors (Ayala et al., 1973, Gilpin and Ayala, 1973 Schoener, 1976 Goh and Agnew, 1977, Gallagher et al., 1990), but never for mutualism (but see a generalized Verhulst-Lotka-Volterra model in Ribeiro et al. 2014 and a specific facultative-obligate model in Wang 2016). Given the prevalence of nonlinear intraspecific density dependence, and its known influence on dynamics in other ecological contexts, the dearth of mutualism models that assume anything besides logistic growth suggests that our understanding of mutualistic dynamics may be quite incomplete.

In sum, the Lotka-Volterra mutualism model makes two separate assumptions that are likely violated in many natural systems: a linear effect of mutualistic interactions, and linear intraspecific density dependence. The former is widely thought responsible for the Lotka-Volterra mutualism model's unrealistic behavior, but since the latter has never been investigated in the context of mutualisms, the relative importance of these two simplifying assumptions remains unclear. While we agree that many mutualistic interactions are likely nonlinear, the same could be said of competitive interactions, and yet Lotka-Volterra competition models endure. Is the need to eschew linear interaction rates truly fundamental for mutualisms? We approached this line of inquiry by returning to the original Lotka-Volterra mutualism model. To complement what is already known, we relax the assumption of linear intraspecific density dependence while leaving the assumption of a linear mutualistic functional response intact. We accomplish this by using the $\theta$-logistic equation, which can decelerate or accelerate as a function of intraspecific density. We found that any accelerating model was always stable, and that decelerating models were stable with weak mutualism. We therefore conclude that relaxing either of the Lotka-Volterra model's major simplifying assumptions can prevent unrealistic model behavior. Given that nonlinear intraspecific density dependence appears to be widespread, nonlinearity in mutualistic interaction rates may be less important for stabilizing mutualisms than was previously believed. 


\section{Methods}

The Lotka-Volterra mutualism model for population densities of two species, $N_{1}$ and $N_{2}$, takes the form

$$
\begin{aligned}
& \frac{1}{N_{1}} \frac{d N_{1}}{d t}=f_{1}\left(N_{1}\right)+\beta_{1} N_{2}=r_{1}-\alpha_{1} N_{1}+\beta_{1} N_{2} \\
& \frac{1}{N_{2}} \frac{d N_{2}}{d t}=f_{2}\left(N_{2}\right)+\beta_{2} N_{1}=r_{2}-\alpha_{2} N_{2}+\beta_{2} N_{1} .
\end{aligned}
$$

That is, the per capita change in population $i$ 's density is a function of intraspecific density, $f_{i}\left(N_{i}\right)$, and a linear function of mutualist partner density, $\beta_{i} N_{j}$. It is further assumed that intraspecific density dependence, $f_{i}\left(N_{i}\right)$, is logistic. This means the per capita growth rate approaches $r_{i}$ when $N_{i}$ approaches 0 , and linearly decreases as intraspecific density increases, with slope $-\alpha_{i}$. Assuming positive parameter values, eq. (1) has the following behavior: each population grows when rare, each population has a stable positive abundance in the absence its mutualist partner, a feasible 2species equilibrium exists if $\beta_{i} \beta_{j}<\alpha_{i} \alpha_{j}$, and unbounded exponential growth occurs if $\beta_{i} \beta_{j} \geq \alpha_{i} \alpha_{j}$ (Vandermeer and Boucher, 1978).

The first terms in eq. (1) have not received the same scrutiny as the last terms. We suspect this has more to do with the ubiquity of the logistic model than careful evaluation of its application here. To explore this, we relax the assumption of logistic growth - the assumption that the difference between per capita births and deaths linearly decreases as density increases. We relax this assumption by modeling per capita growth rates using the $\theta$-logistic model. This causes the per capita growth rate to be a decelerating function of density if the exponent $(\theta)$ is $<1$ and an accelerating function if it is $>1$ (Fig. 1). An exponent of 0 yields a density independent model and an exponent of 1 recovers the logistic model. We write each density dependent term, $f_{i}\left(N_{i}\right)$, as $-\alpha_{i} N^{\theta_{i}}$ :

$$
\begin{aligned}
& \frac{1}{N_{1}} \frac{d N_{1}}{d t}=r_{1}-\alpha_{1} N_{1}^{\theta_{1}}+\beta_{1} N_{2} \\
& \frac{1}{N_{2}} \frac{d N_{2}}{d t}=r_{2}-\alpha_{2} N_{2}^{\theta_{2}}+\beta_{2} N_{1} .
\end{aligned}
$$

Our main experiment involved assessing stability of eq. (2) by modifying the four types of intraspecific density dependence (density independent, decelerating, linear, accelerating) in a model of mutualism with a linear functional response. Additionally, in the Supplementary Material, we (1) modeled per capita birth and death rates as separate nonlinear functions, each with their own exponent, (2) considered when exponents are different between the two populations, and (3) used a saturating functional response instead of a linear one using the procedures described in the re- 
mainder of this section. A combination of analytical, numerical, and graphical techniques were used to assess the behavior of eq. (2). Specifically, we (i) found equilibria and (ii) determined the behavior around each equilibrium using local stability analysis. When analytical solutions were not possible (i.e., $\theta_{i} \neq 0$ or 1 ), we solved for stable equilibria numerically using the Livermore Solver for Ordinary Differential Equations, Automatic (LSODA) (Hindmarsh, 1983 , Petzold, 1983) and solved for unstable equilibria using Newton's method. LSODA is an integrator that was used because of its generality and ability to automatically handle stiff and non-stiff initial value problems, which were properties of our models. Newton's method is an iterative root-finding algorithm we used to find unstable equilibria to a precision of $10^{-15}$, across state-space, from $N_{i}=0-10^{100}$ by orders of 10. Analyses were conducted in the R language and environment ( $\mathrm{R}$ Core Team, 2016), with LSODA implemented in the deSolve package (Soetaert et al., 2010; Soetaert, 2010) and Newton's method in the rootSolve package (Soetaert and Herman, 2009, Soetaert, 2009). Graphical analyses were conducted using a modified version of the $\mathrm{R}$ package phase R (Grayling, 2014). Specifically, phase plots were created, using direction fields and zero-growth isoclines (i.e., nullclines) to corroborate and visualize our numerical findings. Code to run our analyses can be found at https://github.com/dispersing/Mutualism-NonlinearDensityDependence

Parameter values for numerical analyses focused on the type of nonlinear per capita intraspecific density dependence (i.e., $\theta_{i}$ ) and the strength of mutualism (i.e., $\beta_{i}$ ). For both of these types of parameters, we considered values ranging from $10^{-2}-10^{2}$. The other parameter values $\left(r_{i}\right.$ and $\left.\alpha_{i}\right)$ did not qualitatively affect the model behavior in terms of number or stability of equilibria (C. Moore, unpublished results), so we do not discuss their effects in detail.

\section{Results}

General results. For all analyses with linear functional responses we found between 3 and 5 nonnegative equilibrium population sizes (Fig. 2). Analytically, we found that $(0,0)$ was always an equilibrium and always unstable. Further, there were always two boundary equilibria $\left(N_{1}>0,0\right)$ and $\left(0, N_{2}>0\right)$, both of which were saddle nodes. The instability of the trivial and boundary equilibria means that populations always grow when rare, as expected. Numerically, we found that in cases where interior equilibria were present $\left(N_{1}^{*}>0, N_{2}^{*}>0\right)$, there were either one or two points. In cases where there was only one equilibrium point, it was always stable; in cases where there were two equilibrium points, the point proximal to the origin $(0,0)$ was always stable and the point distal to the origin was a saddle node. Fig. 3 shows the six qualitatively different types of phase planes 
found in this study: (i) a trivial density independent case $\theta_{i}=0$; (ii \& iii) unstable and stable configurations when intraspecific density dependence was decelerating, $0<\theta_{i}<1$; (iv \& v) unstable and stable configurations when intraspecific density dependence was linear, $\theta_{i}=1$; and (vi) a stable configuration when intraspecific density dependence was accelerating, $\theta_{i}>1$.

In general, in the absence of mutualism, decelerating intraspecific density dependence increased both species' densities at equilibrium $\left(\beta_{i}=0\right.$ plane in Fig. 4 , left panel). Oppositely, accelerating intraspecific density dependence decreased the equilibrium densities. Strong mutualism (high $\beta_{i}$ ) destabilized populations with decelerating intraspecific density dependence, but populations with accelerating intraspecific density dependence were always stable (Fig. 4, center panel; note that only stable equilibria are shown, so missing portions of the surface at high $\beta_{i}$ and low $\theta_{i}$ denote loss of stability; see also Supplemental Material, section 2). Further, when a stable interior equilibrium was present, adding mutualism to populations with decelerating intraspecific density dependence generated a larger benefit of mutualism than with accelerating intraspecific density dependence (Fig. 4 , right panel).

Decelerating density dependence, $0<\theta_{i}<1$. When $0<\theta_{i}<1$, we found that there were $1-2$ interior equilibria (3-5 total equilibria), depending on the strength of mutualism. In the absence of mutualism, the interior equilibrium (and consequently the boundary equilibria by setting either coordinate to 0 ) is at

$$
\left(\left(\frac{r_{1}}{\alpha_{1}}\right)^{\theta_{1}^{-1}},\left(\frac{r_{2}}{\alpha_{2}}\right)^{\theta_{2}^{-1}}\right)
$$

Notice the $\theta_{i}^{-1}$ exponent. In these cases of decelerating density dependence, as $\theta_{i}$ decreases from 1 , the greatest change in growth rate occurs at lower densities (Fig. 1). Furthermore, the equilibrium density in the absence of mutualism grows larger as $\theta_{i}$ decreases.

Adding mutualism to populations with decelerating density dependence changed the dynamics in either of two ways: (i) it destabilized the populations resulting in unbounded population growth (Fig. 3 top-center panel) or (ii) it created both a stable and saddle node (Fig. 3 , top-right panel). For very small values of $\theta_{i}$, populations were always unstable with mutualism added (i.e., $\beta_{i}>0$ ). As decelerating density dependence became more linear (i.e., as $\theta_{i} \rightarrow 1$ ), however, weak mutualism (small values of $\beta_{i}$ ) resulted in an alternative configuration in which zero-growth isoclines crossed twice. Of these two equilibria, the stable equilibrium point was always larger than in the absence of mutualism $\left(\beta_{i}=0\right)$ and the saddle node was always larger than the stable point. For the same values of $\theta_{i}$ with stable and saddle nodes, increasing $\beta_{i}$ increased the stable point and decreased 
the saddle point. Continuing to increase $\beta_{i}$ ultimately resulted in a saddle-node bifurcation, beyond which all configurations were unstable, illustrated as the light-dark gray boundary in Fig. 2 .

Linear density dependence, $\theta_{i}=1$. When $\theta_{i}=1$, there were either 0 or 1 interior equilibrium configurations (3 or 4 total equilibria) that respectively corresponded to the absence of presence of an interior stable point. Linear density dependence is equivalent to the most traditional formulation of mutualism, the Lotka-Volterra competition model with the sign reversed of the effect of another population. Although the behavior of this model is well-known, we summarize its properties briefly here for ease of comparison. In the absence of mutualism, the interior equilibrium (and consequently the boundary equilibria by setting either value to 0 ) is at

$$
\left(\frac{r_{1}}{\alpha_{1}}, \frac{r_{2}}{\alpha_{2}}\right)
$$

The slope of the zero-growth isocline as it increases from the boundary equilibrium is $\frac{\beta_{i} N_{j}}{\alpha_{i}}$, and zero-growth isoclines form a stable interior equilibrium point anytime $\beta_{i} \beta_{j}<\alpha_{i} \alpha_{j}$. This is equivalent to the more traditional notation, $\alpha_{i j} \alpha_{j i}<\alpha_{i i} \alpha_{j j}$ found in ecology texts (e.g., May, 1981, DeAngelis et al. 1986, Kot, 2001). The location of the stable interior equilibrium point is

$$
\left(\frac{r_{1}}{\alpha_{1}}+\frac{\beta_{1} r_{1}\left(\beta_{1}+\alpha_{1}\right)}{\alpha_{1}^{2}\left(\alpha_{1}-\beta_{1}^{2}\right)}, \frac{r_{2}}{\alpha_{2}}+\frac{\beta_{2} r_{2}\left(\beta_{2}+\alpha_{2}\right)}{\alpha_{2}^{2}\left(\alpha_{2}-\beta_{2}^{2}\right)}\right)
$$

Accelerating density dependence, $\theta_{i}>1$. When $\theta_{i}>1$, there was always one interior equilibrium $(4$ total equilibria), irrespective of the strength of mutualism (Figs. 2, 4). In the absence of mutualism, the interior equilibrium is again given by (3). Again, note the $\theta_{i}^{-1}$ in the exponent. In these cases of accelerating density dependence, as $\theta_{i}$ increase from 1 , the greatest change in growth rate occurs at higher densities (Fig. 1). Furthermore, the equilibrium point in the absence of mutualism decreases as $\theta_{i}$ increased (Fig. 4 , left panel). With mutualism $\left(\beta_{i}>0\right)$, in addition to always being stable, the benefit decreased as $\theta_{i}$ increased.

Supplementary Material: Births and deaths as separate processes, interspecific differences in intraspecific density dependence, and saturating functional response. Assuming per capita birth and death rates were independent processes, we modeled them as separate nonlinear functions. Our main finding was that as long as one of the exponents was accelerating, the interior equilibrium point would always be stable. We found no qualitatively new model behaviors when we allowed the two species to have differently shaped intraspecific density dependent functions. Notably, the pres- 
ence of a single, stable interior equilibrium point is possible as long as either species has accelerating density dependence. We also replaced the linear functional response in eq. (2) with a saturating functional response, finding that the saturating function would always stabilize the interior equilibrium point, but with less mutualistic benefit (the difference in density in the absence and presence of a mutualist partner).

\section{Discussion}

Lotka-Volterra models of mutualism assume that intraspecific density linearly decreases per capita growth rates. Other population models of mutualism have inherited this assumption and have generally concluded that 2 -species models of mutualism are inherently unstable. In real populations, however, not only do nonlinear per capita growth rates exist, but they seem to be the rule rather than the exception (Stubbs, 1977; Fowler, 1981; Sibly et al., 2005). In this study, we examined how relaxing the assumption of linearly dependent per capita growth rates affected stability and mutualistic benefit in these models. We found that when per capita growth rates decrease most strongly at low densities and are decelerating, mutualism usually destabilizes the model. In contrast, when growth rates decrease most strongly at high densities and are accelerating, models are always stable with mutualism. Despite the tendency for mutualism to destabilize the 2-species equilibrium with decelerating density dependence, the benefit was greater compared to stabilizing, accelerating density dependence.

Our paper presents an alternative way that the classic Lotka-Volterra mutualism model can be modified to stabilize mutualism. Simply put, we added a layer of biological realism (nonlinear intraspecific density dependence) to the Lotka-Volterra mutualism model and we found informative ways that within-species properties could stabilize mutualism, even with a linear functional response modeling the interaction between species. Support for decelerating and accelerating density dependence has largely been based on large datasets from observational studies (e.g., 1750 species of mammals, birds, fish, and insects in Sibly et al. 2005). Further work to determine whether species that engage in mutualism are more likely to have accelerating intraspecific density dependence, which we found to be stabilizing, would be useful.

From an ecological perspective, species' nonlinear responses to intraspecific density arise from differences in ecological habits or population structure. Sedentary organisms, like many plants for example, exhibit a more-or-less-constant death rate at low-to-intermediate population densities, and then at higher densities death rates tend to rapidly increase (as in scramble competition or 
self-thinning, Yoda et al. 1963) or increase linearly (as in contest competition, Crawley and Ross, 1990), resulting in accelerating density dependence. Subsets of populations, such as age or stage, can experience different vital rates and generate nonlinear density dependence for populations as a whole. In African ungulates, for example, increases in density led to increases in adult mortality, while juvenile mortality remained relatively constant at all population densities (Owen-Smith, 2006). In fact, many mutualisms occur between species with structured populations, so our study may lend insights into these interactions. As examples, many plant-mycorrhizal associations are mutualistic in the seedling stage (Grime et al., 1987, Jones and Smith, 2004, van der Heijden and Horton, 2009) and adult plants engage in mutualistic interactions with pollinators when they reach a reproductive stage.

From an evolutionary perspective, a long-standing theory about why we see nonlinear density dependence comes from evolutionary theories of life-history strategies; i.e., $r$ - and $K$-selected species (Gilpin and Ayala, 1973, Stubbs, 1977, Fowler, 1981), including $\theta$-selection (Gilpin et al., 1976). Setting aside historical controversies, this body of theory has generated very useful quantities like the specific growth rate of a population. The most general prediction made is that populations with a high specific growth rate (commonly referred to as $r$-selected) should exhibit decelerating density dependence since their survival probability drops off precipitously at relatively low densities. On the other hand, populations with a low specific growth rate (commonly referred to as $K$-selected) should exhibit accelerating density dependence since their survival probabilities drop off at relatively high densities (see Figs. 1, 2 in Fowler, 1981). Based on our study we suspect that different life-history strategies may both be a result of and a causative factor in the evolution of mutualistic interactions, and further work should examine how engaging in a mutualistic interaction should change the shape of density dependence and how changing density dependence affects a species ability to engage in a mutualistic interaction.

In conclusion, the linear functional response has historically been the scapegoat for theoretical studies of the population dynamics of mutualism. For example, the eminent Lord Robert May (1976) writes:

...the simple, quadratically nonlinear, Lotka-Volterra models ... are inadequate for even a first discussion of mutualism, as they tend to lead to silly solutions in which both populations undergo unbounded exponential growth, in an orgy of mutual benefaction. Minimally realistic models for two mutualists must allow for saturation in the magnitude of at least one of the reciprocal benefits. 
In this paper, we build on May's idea of modifying the Lotka-Volterra mutualism model; not through the saturation of benefits, but through intraspecific density dependence. We found that biologicallyrealistic nonlinear density dependence significantly changes the dynamics of the original LotkaVolterra mutualism model, where we found that accelerating density dependence always stabilized our models but with weaker mutualistic benefit relative to decelerating density dependence. We hope that this study will further stimulate ecologists to consider all simplifying of assumptions of even the most basic models and also to investigate more deeply into the relationships between intraspecific density, interspecific density, and population growth to gain a better grasp on mutualistic population dynamics.

\section{Acknowledgements}

We thank Katie Dixon, Frances Ji, Brian Lerch, Robin Snyder, and Chris Steiha for comments on an early draft of the manuscript. K.C.A. and C.M.M. were supported in part by a James S. McDonnell Foundation Complex Systems Scholar award to K.C.A. 


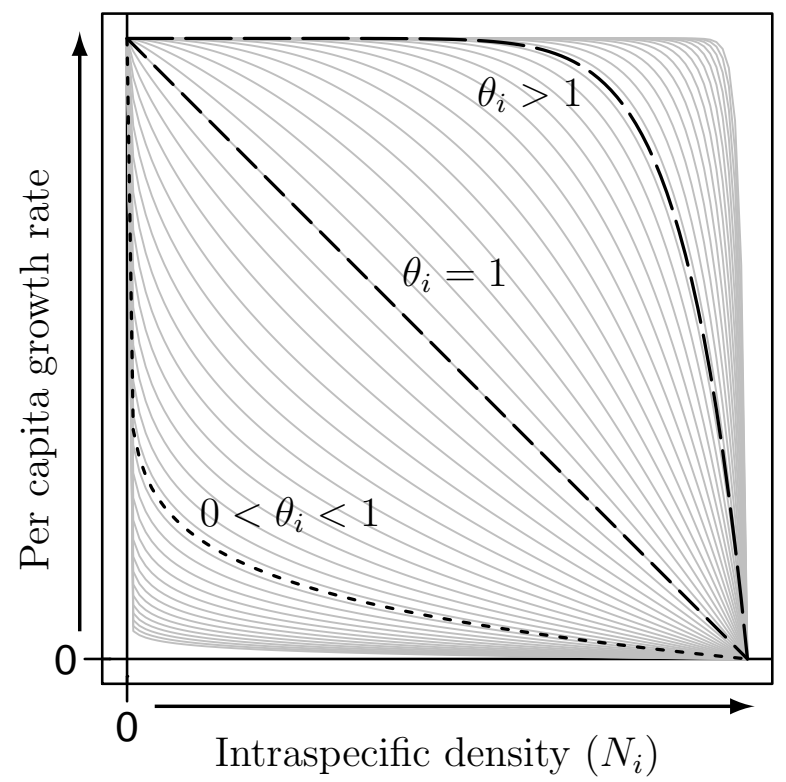

Figure 1: Values of $\theta_{i}$ used in eq. (2) to represent nonlinear per capita growth rates before accounting for the effects of mutualism. The figure shows how the per capita growth rates change as a function of intraspecific density, $N_{i}$. The actual values used for numerical analyses are presented in light gray, with highlighted examples of decelerating intraspecific density dependence $\left(\theta_{i}=1 / 10\right.$; short dashes, $\left.\cdots \cdot\right)$, linear intraspecific density dependence $\left(\theta_{i}=1\right.$; medium dashes, $-\mathbf{-})$, and accelerating intraspecific density dependence $\left(\theta_{i}=10\right.$; long dashes, $\left.-\mathbf{C}\right)$. 


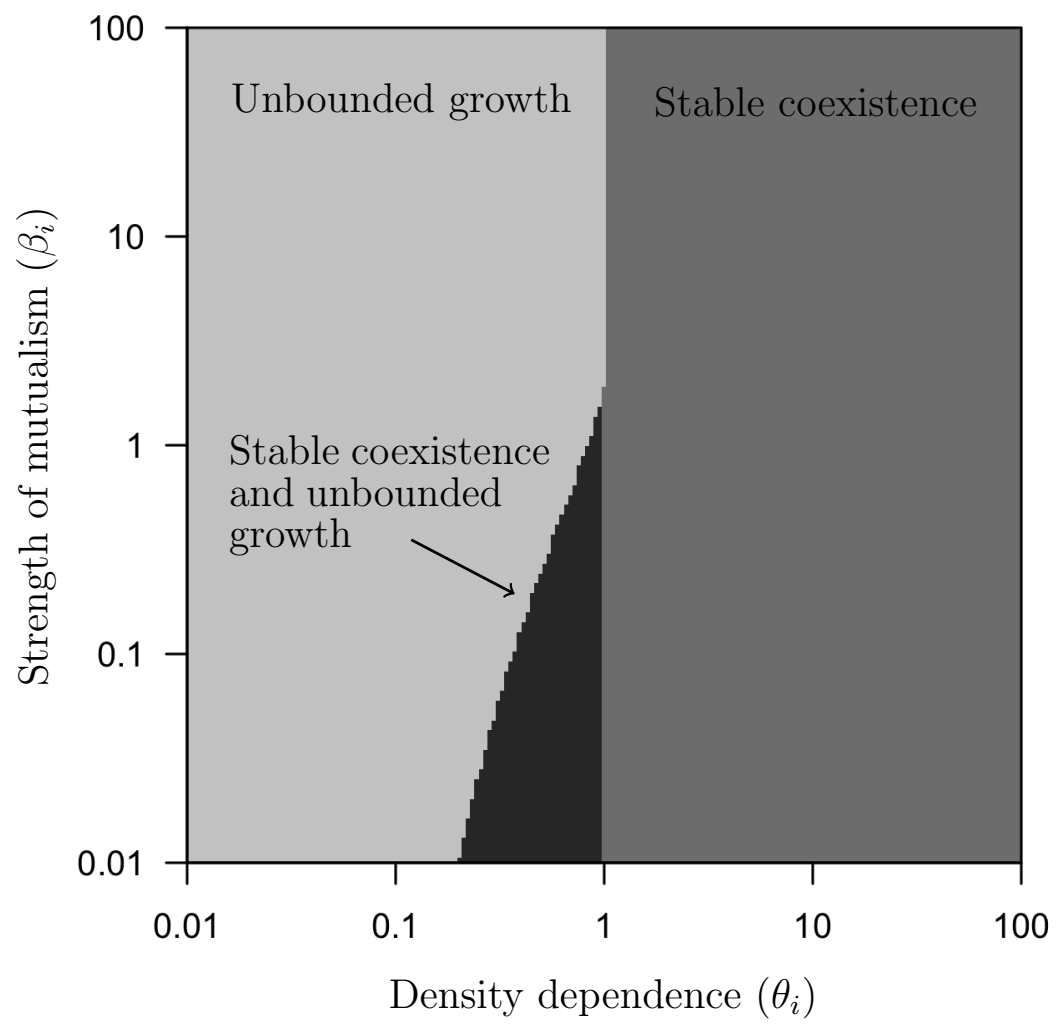

Figure 2: Number of equilibrium points (shades of gray) across all values of intraspecific density dependence $\left(\theta_{i}\right)$ and strength of mutualism $\left(\beta_{i}\right)$, while holding the remaining parameters constant at $r_{i}=4, \alpha_{i}=2$. Across all analyses, there were always between 1 and 2 interior equilibria ( 3 and 5 total equilibria, including the trivial and boundary equilibria). The light-gray regions corresponds to unstable configurations where no interior equilibrium existed, the medium-gray regions correspond to stable configurations where one stable interior equilibrium existed, and the dark-gray regions correspond to areas with two interior equilibria, one stable at low densities and one saddle at high densities. 
bioRxiv preprint doi: https://doi.org/10.1101/108175; this version posted November 18, 2017. The copyright holder for this preprint (which was not certified by peer review) is the author/funder, who has granted bioRxiv a license to display the preprint in perpetuity. It is made available under aCC-BY-NC 4.0 International license.
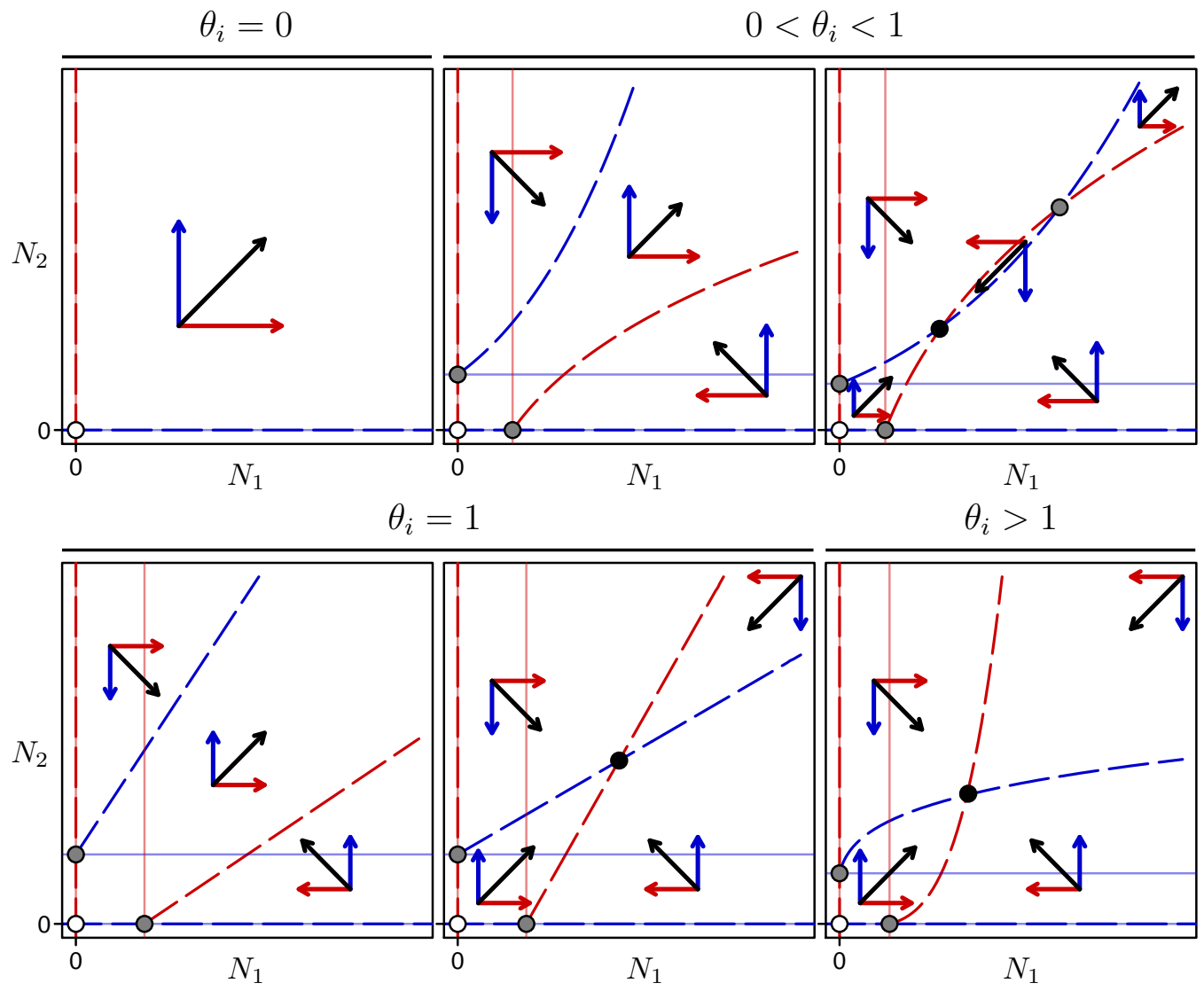

Figure 3: Phase planes representing the qualitative dynamics of 2-species mutualistic interactions for different models of per capita intraspecific density dependence. Each panel shows the densities of $N_{1}$ and $N_{2}$ on the $x$ - and $y$-axes. Within each panel, zero-growth isoclines (nullclines) are shown for $N_{1}$ (red) and $N_{2}$ (blue): (i) when there is no mutualism $\left(\beta_{i}=0\right)$ as solid, light lines $(-$ or -$)$ and (ii) when mutualism is present $\left(\beta_{i}>0\right)$ as dashed lines $\left(--\right.$ or -- ). Arrows within panels show the qualitative direction vectors for $N_{1}$ (red), $N_{2}$ (blue), and together (black) for all changes in direction for each phase plane. Points within panels represent unstable (white), stable (black), or saddle nodes (gray). The trivial intraspecific density independent result $\left(\theta_{i}=0\right)$ is shown in the top-left panel, the two results of decelerating intraspecific density dependence $\left(0<\theta_{i}<1\right)$ are shown in the top-center and -right panels, linear intraspecific density dependence $\left(\theta_{i}=1\right)$ is shown in the bottom-left and -center panels, and accelerating intraspecific density dependence $\left(\theta_{i}>1\right)$ is shown in the bottom-right panel. 

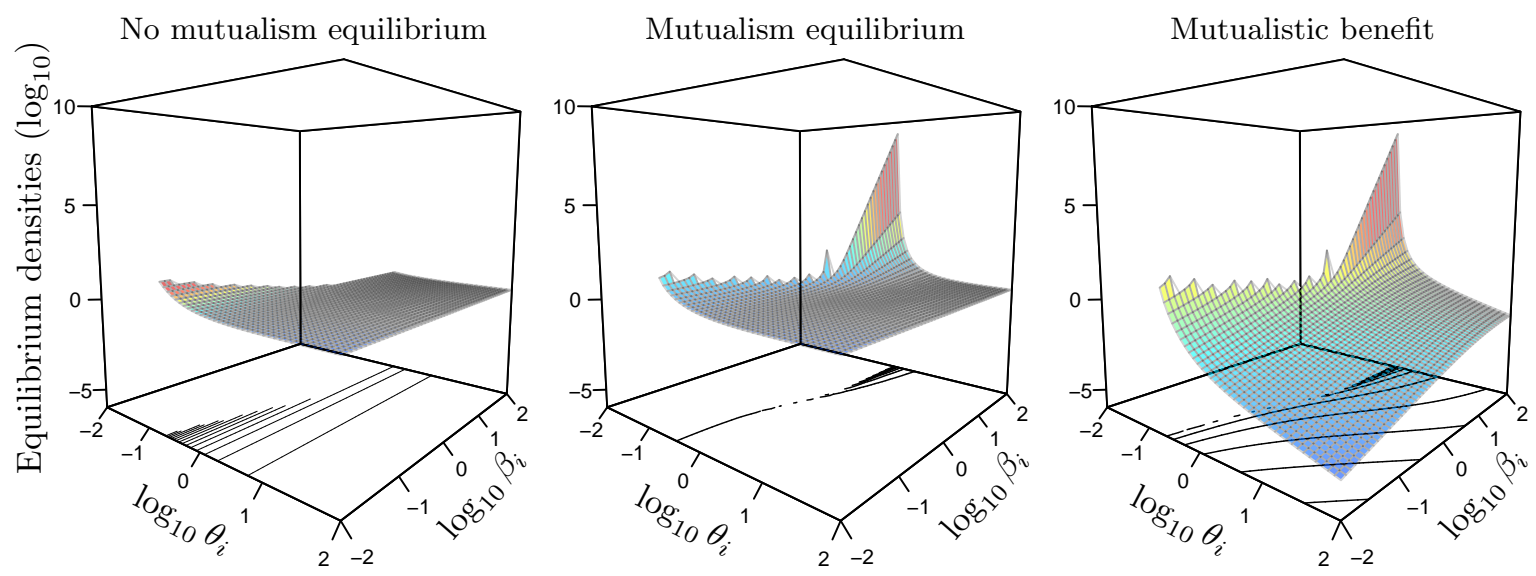

Figure 4: For model 22, nonlinear per capita growth rates with a linear functional response of mutualism, the location of the interior equilibrium in the absence of mutualism $\left(\beta_{i}=0\right.$, left), stable interior equilibrium with mutualism (center), and the benefit of mutualism as the difference between the two (right). The locations of equilibria were identified as the Euclidean distance from the origin, $\sqrt{\left(N_{i}^{*}\right)^{2}+\left(N_{j}^{*}\right)^{2}}$, for identical parameters for each species: $r_{i}=4, \alpha_{i}=2$. Each panel shows the aforementioned response on the vertical axis, the type of intraspecific density dependence $\left(\theta_{i}\right.$ from $\left.10^{-2}-10^{2}\right)$ on the left horizontal axis, and the strength of mutualism $\left(\beta_{i}\right.$ from $\left.10^{-2}-10^{2}\right)$ on the right horizontal axis. Additionally, each panel shows the relative values of each surface (colors), the absolute values of each surface (same axes across panels), and contour lines at the base of each plot show changes in the surface. Further, in areas where there is no surface, there was no stable interior equilibrium when $\beta_{i} \neq 0$ (center). In the left panel without mutualism, there were stable interior equilibria across all values of $\theta_{i}$, but we removed the same part of the surface to aid comparison across panels. 
Albrecht, F., Gatzke, H., Haddad, A., Wax, N., 1974. The dynamics of two interacting populations. Journal of Mathematical Analysis and Applications 46 (3), 658-670.

Ayala, F. J., Gilpin, M. E., Ehrenfeld, J. G., 1973. Competition between species: Theoretical models and experimental tests. Theoretical Population Biology 4 (3), 331-356.

Baker, A. C., 2003. Flexibility and specificity in coral-algal symbiosis: Diversity, ecology, and biogeography of symbiodinium. Annual Review of Ecology, Evolution, and Systematics 34, 661-689.

Brodo, I. M., Sharnoff, S. D., Sharnoff, S., 2001. Lichens of North America. Yale University Press.

Bronstein, J. L. (Ed.), 2015. Mutualism. Oxford University Press.

Christiansen, F. B., Fenchel, T. M., 1977. Theories of Populations in Biological Communities. Ecological Studies 20. Springer-Verlag Berlin.

Coulson, T., Ezard, T. H. G., Pelletier, F., Tavecchia, G., Stenseth, N. C., Childs, D. Z., Pilkington, J. G., Pemberton, J. M., Kruuk, L. E. B., Clutton-Brock, T. H., Crawley, M. J., 2008. Estimating the functional form for the density dependence from life history data. Ecology 89 (6), 1661-1674.

Crawley, M. J., Ross, G. J. S., 11 1990. The population dynamics of plants. Philosophical Transactions of the Royal Society of London. Series B: Biological Sciences 330 (1257), 125.

DeAngelis, D. L., Post, W. M., Travis, C. C., 1986. Positive feedback in natural systems. Vol. 15 of Biomathematics. Springer-Verlag, Berlin.

Fowler, C. W., 1981. Density dependence as related to life history strategy. Ecology 62 (3), 602-610.

Gallagher, E. D., Gardner, G. B., Jumars, P. A., 1990. Competition among the pioneers in a seasonal soft-bottom benthic succession: field experiments and analysis of the Gilpin-Ayala competition model. Oecologia 83 (4), 427-442.

Gause, G. F., Witt, A. A., 1935. Behavior of mixed populations and the problem of natural selection. American Naturalist, 596-609.

Gilpin, M. E., Ayala, F. J., 12 1973. Global models of growth and competition. Proceedings of the National Academy of Sciences 70 (12), 3590-3593.

Gilpin, M. E., Case, T. J., Ayala, F. J., 1976. $\theta$-selection. Mathematical Biosciences 32 (1), 131-139.

Goh, B. S., Agnew, T. T., 1977. Stability in Gilpin and Ayala's models of competition. Journal of Mathematical Biology 4 (3), 275-279. 
Grayling, M. J., 2014. phaseR: Phase Plane Analysis of One and Two Dimensional Autonomous ODE Systems. R package version 1.3.

Grime, J. P., Mackey, J. M. L., Hillier, S. H., Read, D. J., 1987. Floristic diversity in a model system using experimental microcosms. Nature 328 (6129), 420-422.

Hindmarsh, A. C., 1983. ODEPACK, A systematized collection of ODE solvers. In: Stepleman, R. S. (Ed.), Scientific Computing. North-Holland, Amsterdam, pp. 55-64.

Holland, J. N., DeAngelis, D. L., May 2010. A consumer-resource approach to the density-dependent population dynamics of mutualism. Ecology 91 (5), 1286-1295.

Holland, J. N., DeAngelis, D. L., Bronstein, J. L., Mar 2002. Population dynamics and mutualism: Functional responses of benefits and costs. American Naturalist 159 (3), 231-244.

Howe, H. F., Smallwood, J., 1982. Ecology of seed dispersal. Annual Review of Ecology, Evolution, and Systematics 13, 201-228.

Jones, M. D., Smith, S. E., 2004. Exploring functional definitions of mycorrhizas: are mycorrhizas always mutualisms? Canadian Journal of Botany 82 (8), 1089-1109.

Kiers, E. T., Rousseau, R. A., West, S. A., Denison, R. F., 09 2003. Host sanctions and the legumerhizobium mutualism. Nature 425 (6953), 78-81.

Kot, M., 2001. Elements of mathematical ecology. Cambridge University Press.

Levey, D. J., Silva, W. R., Galetti, M., 2002. Seed dispersal and frugivory: ecology, evolution, and conservation. CABI.

May, R. M., 1976. Models for two interacting populations. In: Theoretical ecology: Principles and applications, 1st Edition. Sinauer Associates, Inc.

May, R. M., 1981. Models for two interacting populations. In: Theoretical ecology: Principles and applications, 2nd Edition. Sinauer Associates, Inc.

Owen-Smith, N., 2006. Demographic determination of the shape of density dependence for three african ungulate populations. Ecological Monographs 76 (1), 93-109.

Petzold, L., 1983. Automatic selection of methods for solving stiff and nonstiff systems of ordinary differential equations. SIAM journal on scientific and statistical computing 4 (1), 136-148. 
Pomerantz, M. J., Thomas, W. R., Gilpin, M. E., 1980. Asymmetries in population growth regulated by intraspecific competition: Empirical studies and model tests. Oecologia 47 (3), 311-322.

R Core Team, 2016. R: A Language and Environment for Statistical Computing. R Foundation for Statistical Computing, Vienna, Austria, R version 3.2.4 (2016-03-10)— "Very Secure Dishes" Edition.

URL https : //www.R-project .org/

Revilla, T. A., Aug 2015. Numerical responses in resource-based mutualisms: A time scale approach. Journal of Theoretical Biology 378, 39-46.

Ribeiro, F., Cabella, B. C. T., Martinez, A. S., 2014. Richards-like two species population dynamics model. Theory in Biosciences 133 (3), 135-143.

URL http://dx .doi .org/10.1007/s12064-014-0205-z

Richards, F. J., 06 1959. A flexible growth function for empirical use. Journal of Experimental Botany 10 (2), 290-301.

Rico-Gray, V., Oliveira, P. S., 2007. The ecology and evolution of ant-plant interactions. University of Chicago Press.

Schoener, T. W., 1973. Population growth regulated by intraspecific competition for energy or time: Some simple representations. Theoretical Population Biology 4 (1), 56-84.

Schoener, T. W., 1976. Alternatives to Lotka-Volterra competition: Models of intermediate complexity. Theoretical Population Biology 10 (3), 309-333.

Sibly, R. M., Barker, D., Denham, M. C., Hone, J., Pagel, M., 07 2005. On the regulation of populations of mammals, birds, fish, and insects. Science 309 (5734), 607.

Smith, D. W., Cooper, S. D., 1982. Competition among cladocera. Ecology, 1004-1015.

Smith, F. E., 1963. Population dynamics in Daphnia magna and a new model for population growth. Ecology 44 (4), 651-663.

Soetaert, K., 2009. rootSolve: Nonlinear root finding, equilibrium and steady-state analysis of ordinary differential equations. $\mathrm{R}$ package 1.6 .

Soetaert, K., 2010. deSolve: Solvers for Initial Value Problems of Differential Equations (ODE, DAE, DDE). Version 1.14. 
Soetaert, K., Herman, P. M., 2009. A Practical Guide to Ecological Modelling. Using R as a Simulation Platform. Springer.

Soetaert, K., Petzoldt, T., Setzer, R. W., 2010. Solving differential equations in R: Package deSolve. Journal of Statistical Software 33 (9), 1-25.

Sprent, J. I., Sutherland, J. M., de Faria, S. M., Dilworth, M. J., Corby, H. D. L., Becking, J. H., Materon, L. A., Drozd, J. W., 1987. Some aspects of the biology of nitrogen-fixing organisms. Philosophical Transactions of the Royal Society of London. Series B, Biological Sciences 317 (1184), $111-129$.

Stadler, B., Dixon, A. F. G., 2008. Mutualism: ants and their insect partners. Cambridge University Press.

Stubbs, M., 1977. Density dependence in the life-cycles of animals and its importance in $K$ - and r-strategies. Journal of Animal Ecology 46 (2), 677-688.

Turchin, P., 2003. Complex population dynamics: a theoretical/empirical synthesis. Vol. 35. Princeton University Press.

van der Heijden, M. G. A., Horton, T. R., 2009. Socialism in soil? the importance of mycorrhizal fungal networks for facilitation in natural ecosystems. Journal of Ecology 97 (6), 1139-1150.

van der Heijden, M. G. A., Martin, F. M., Selosse, M.-A., Sanders, I. R., 2015. Mycorrhizal ecology and evolution: the past, the present, and the future. New Phytologist 205 (4), 1406-1423.

Vandermeer, J. H., Boucher, D. H., 1978. Varieties of mutualistic interaction in population models. Journal of Theoretical Biology 74 (4), 549-558.

Wang, D., 2016. Dynamic behaviors of an obligate Gilpin-Ayala system. Advances in Difference Equations 2016 (1), 270.

Willmer, P. G., 2011. Pollination and floral ecology. Princeton University Press.

Wolin, C. L., 1985. The population dynamics of mutualistic systems. In: Boucher, D. H. (Ed.), The biology of mutualism. Oxford University Press, Inc.

Wright, D. H., 1989. A simple, stable model of mutualism incorporating handling time. American Naturalist 134 (4), 664-667. 

available under aCC-BY-NC 4.0 International license.

373 Yoda, K., Kira, T., Ogawa, H., Hozumi, K., 1963. Self-thinning in overcrowded pure stands under cultivated and natural conditions. (Intraspecific competition among higher plants XI). Journal of Biology, Osaka City University 14, 107-129. 